\title{
CrystEngComm
}

\section{Polymorphism and polymerisation of acrylic and methacrylic acid at high pressure $\dagger$}

\author{
Iain D. H. Oswald* and Andrew J. Urquhart \\ Received 8th March 2011, Accepted 10th May 2011 \\ DOI: 10.1039/c1ce05295k
}

\begin{abstract}
The polymorphism and polymerisation of two related acids have been investigated under high pressure conditions. Acrylic acid crystallises as a new polymorph at $0.65 \mathrm{GPa}$ whilst methacrylic acid crystallises in a new polymorph at a higher pressure of $1.5 \mathrm{GPa}$. Both these new polymorphs exhibit similar hydrogen bonding motifs to the low temperature phases, however, the molecular packing differs significantly.
\end{abstract}

Polyacrylic acid (PAA) and polymethacrylic acid (PMA) are used in a wide range of research and industrial applications, ranging from superabsorbent materials to drug delivery vehicles. Traditionally PAA and PMA are synthesised using both thermal and photopolymerisation methods from the corresponding monomers acrylic and methacrylic acid. Over the past ten years, Raman spectroscopy has been used to investigate the polymerisation of simple unsaturated compounds such as acrylamide ${ }^{1}$ and ethylene ${ }^{2}$ through the application of pressure. Further studies have investigated the effect of pressure and laser irradiation on compounds such as acetylene, butadiene and propene. ${ }^{3}$ Recently Murli and Song used Raman Spectroscopy to investigate the application of pressure to a monomer capable of hydrogen bonding, acrylic acid. Murli and Song investigated the induced initiator-free polymerization of acrylic acid by compressing pure acrylic acid to approximately $8 \mathrm{GPa}^{4}$ They observed two crystalline phases (I and II) of acrylic acid with Phase I representing a low-temperature configuration whilst Phase II was suggested to enhance molecular interactions towards polymerisation, albeit that the crystal structure for this phase was not reported. Murli and Song stated that 'In situ high-pressure $X$-ray or neutron diffraction measurements would be helpful to monitor the changes in bond lengths of acrylic acid as a function of compression... ${ }^{4}$ With this in mind, we have undertaken to characterise the crystal structures of acrylic acid (A) and its derivative methacrylic acid (B) (Scheme 1) under high-

Strathclyde Institute of Pharmacy and Biomedical Sciences, University of Strathclyde, 161 Cathedral Street, G4 ORE Glasgow, UK. E-mail: iain. oswald@strath.ac.uk; Fax: +44(0)141 552 2562; Tel: +44(0)141 548 2157

$\dagger$ Electronic supplementary information (ESI) available: The electronic supplementary information contains experimental details including crystallographic information as well as further Raman spectra. CCDC reference numbers 816976-816980. For ESI and crystallographic data in CIF or other electronic format see DOI: $10.1039 / \mathrm{clce} 05295 \mathrm{k}$ pressure conditions using X-ray single-crystal diffraction in order to provide further insight into this system.

The low-temperature structure of acrylic acid has previously been determined by Higgs and Sass ${ }^{5}$ and later by Boese et al. ${ }^{6}$ This structure is composed of planes of acrylic acid dimers in space group Ibam. The planes are displaced by $7.705 \AA$ along the $\left[\begin{array}{lll}1 / 2 & 1 / 2 & 0\end{array}\right]$ direction with an interlayer separation of $3.103 \AA(1 / 2$ the $c$-axis length). The packing of the molecules follows an ABAB arrangement with the carboxylic acid groups situated above one another (Fig. 1).

Our initial investigation of the high-pressure structural behaviour of acrylic acid focussed on the use of a Merrill-Bassett Diamond Anvil Cell (DAC) to crystallise the liquid at elevated pressure. ${ }^{7}$ This technique has successfully been used to characterise novel polymorphs of materials, especially those that show a kinetic barrier to conversion on increasing pressure. ${ }^{8}$ By heating the sample in situ at high pressure one is able to overcome this barrier by accessing the new polymorph through the melt. Using this method but with only moderate heating Phase I was isolated at $0.4 \mathrm{GPa}$ (Table S1 $\dagger$ ). The temperature was restricted due to the issue of thermal polymerisation. The cell parameters of Phase I at high pressure and low-temperature are remarkably similar. Interestingly, at high pressure Phase I shows an increase in the interlayer separation over the low-temperature phase determined at $125 \mathrm{~K}$. One might expect that this direction would have been the most compressible as there are no hydrogen bonds along this direction and given its response to temperature $(1.6 \%$ reduction from $138 \mathrm{~K}$ to $125 \mathrm{~K})$. The $b$-direction is the only direction to show any compression and involves a reduction in the intermolecular spacing between the $\mathrm{CH}_{2}$ groups of neighbouring acid dimer (Table S2†).

The growth of single crystals of acrylic acid at higher pressures was not possible as its melting point was too high in order to grow a single crystal directly from the melt. Heating to the usual temperatures of $\sim 423 \mathrm{~K}$ induced polymerisation and so a new technique was required

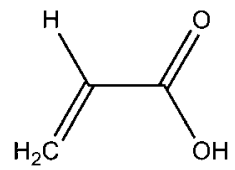

A

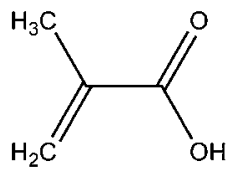

B
Scheme 1 The schematic diagrams of (A) acrylic and (B) methacrylic acids. 


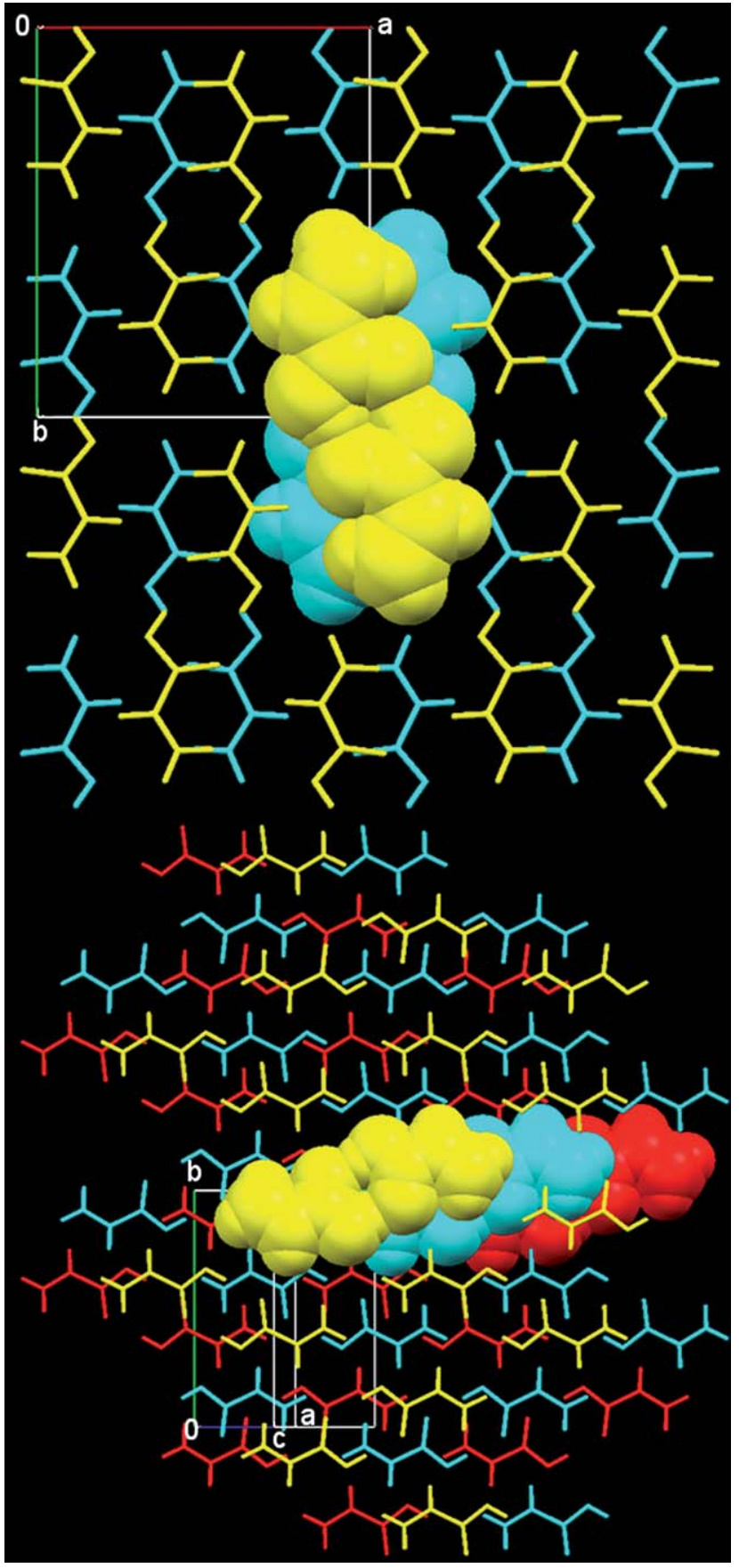

Fig. 1 Packing arrangement of Phase I (upper) and Phase II (lower) of acrylic acid. The colours represent different layers. Note the ABAB packing in Phase I and the ABC packing in Phase II.

in order to explore further the high-pressure behaviour. Using a novel loading technique, inspired by the work of Weck et al. (see ESI $\dagger$ ), ${ }^{9}$ a sample of acrylic acid was crystallised from $4: 1$ methanol : ethanol at a high pressure of $0.65 \mathrm{GPa}$. Using Raman and single-crystal $\mathrm{X}$-ray diffraction this phase was determined to be a novel polymorph of acrylic acid. In order to avoid future confusion with the phases implied by the Raman spectra in the paper of Murli and Song, we are designating this phase, Phase II.

At $0.65 \mathrm{GPa}$, acrylic acid crystallises with the same dimer interaction in monoclinic space group, $P 2_{1} / c$ (see Table S2 $\dagger$ ). Phase II also shows some displacement of the layers but in this instance by $4.161 \AA$ along the [l $\left.\begin{array}{lll}2 & 0 & 1\end{array}\right]$ direction. However, Phase II possesses an ABC packing arrangement with the acid dimer sandwiched between two alkene moieties of the layers above and below (Fig. 1). One of the other major differences is the corrugated nature of the layers in Phase II compared to the planar nature in the other phase. Despite this major difference the separation between discrete dimers remains consistent with the low-temperature phase $(3.107 \AA)$.

In order to verify that the initial product from the high-pressure recrystallisation was the same as the annealed product, the Raman spectrum for both the initial and final products was taken. Fig. 2 shows the fingerprint region of the Raman spectrum for each phase and the change with respect to pressure. One can clearly see the difference in the region of $500-550 \mathrm{~cm}^{-1}$ where there is one main peak at $\sim 520 \mathrm{~cm}^{-1}$ in Phase I whilst there are two distinct peaks in Phase II at $\sim 510$ and $\sim 533 \mathrm{~cm}^{-1}$. The stretch at $\sim 520 \mathrm{~cm}^{-1}$ in Phase I has been previously attributed to the out-of-plane motion of the carboxylic acid group. ${ }^{10}$ Kulbida et al. also observed a weak intensity at $535 \mathrm{~cm}^{-1}$ which they assigned to be the wagging motion of the $\mathrm{CO}_{2}$ moiety; this peak is barely visible as a weak shoulder in the spectrum at $0.2 \mathrm{GPa}\left(\sim 546 \mathrm{~cm}^{-1}\right)$. The intensity of both these peaks is significantly increased and more clearly separated for Phase II.

We attribute this change to the differences in surrounding environment of the $\mathrm{CO}_{2}$ group. The $\mathrm{CO}_{2}$ group in Phase $\mathrm{I}$ is sandwiched between $\mathrm{CO}_{2}$ groups from the layers above and below whilst in Phase II the surrounding $\mathrm{CO}_{2}$ groups are replaced by the alkene moieties. Furthermore the change in topographical nature of the layers may also contribute to the increased motion of the molecule in Phase II.

Comparison of our data against that of Murli and Song shows that they did not observe Phase II during the slow direct compression from ambient pressure to $4.5 \mathrm{GPa}$; this phase was not observed even after three months. However, in further experiment they rapidly compressed the sample to $3.3 \mathrm{GPa}$ obtaining similar data to those reported here, especially in the region between 100 and $200 \mathrm{~cm}^{-1}$ (Fig. S1†).

During the initial attempts to grow a single crystal from the melt it was observed that acrylic acid underwent a reconstructive phase transition if it was heated at a pressure greater than $0.5 \mathrm{GPa}$. If one decompresses this sample one is able to retain this polymorph to 0.38 $\mathrm{GPa}$. Unfortunately, repeated decompression experiments resulted in the melting of the sample and so we were not able to observe whether Phase II was retained to the melting point.

We have also investigated for the first time the structural behaviour of methacrylic acid at both low temperature and high pressure. Methacrylic acid is a liquid at ambient pressure (mp $14{ }^{\circ} \mathrm{C}$ ) and so a crystal was grown from the polycrystalline powder encased in a glass capillary (o.d. $0.3 \mu \mathrm{m}$ ) by cycling the temperature close to the melting point. ${ }^{11}$ The low-temperature phase (Phase I) of methacrylic acid crystallises in monoclinic $P 2_{1} / c$ with the molecules adopting a trans conformation as opposed to the cis confirmation in acrylic acid. This is in line with observations of Badawi et al. who calculated that the trans-form was $\sim 2$ to $3.5 \mathrm{~kJ} \mathrm{~mol}^{-1}$ more stable than the cis-form depending on whether DFT-B3LYP/6-311 $+\mathrm{G}^{* *}$ or MP2/6-311 + G** level of theory was used. ${ }^{12}$ The molecules interact

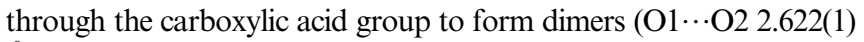
A) (Table S2 $\dagger$ ). The dimers are stacked to form tilted columns along the $a$-direction with a separation between dimers of $3.42 \AA$ (Fig. 3a). 


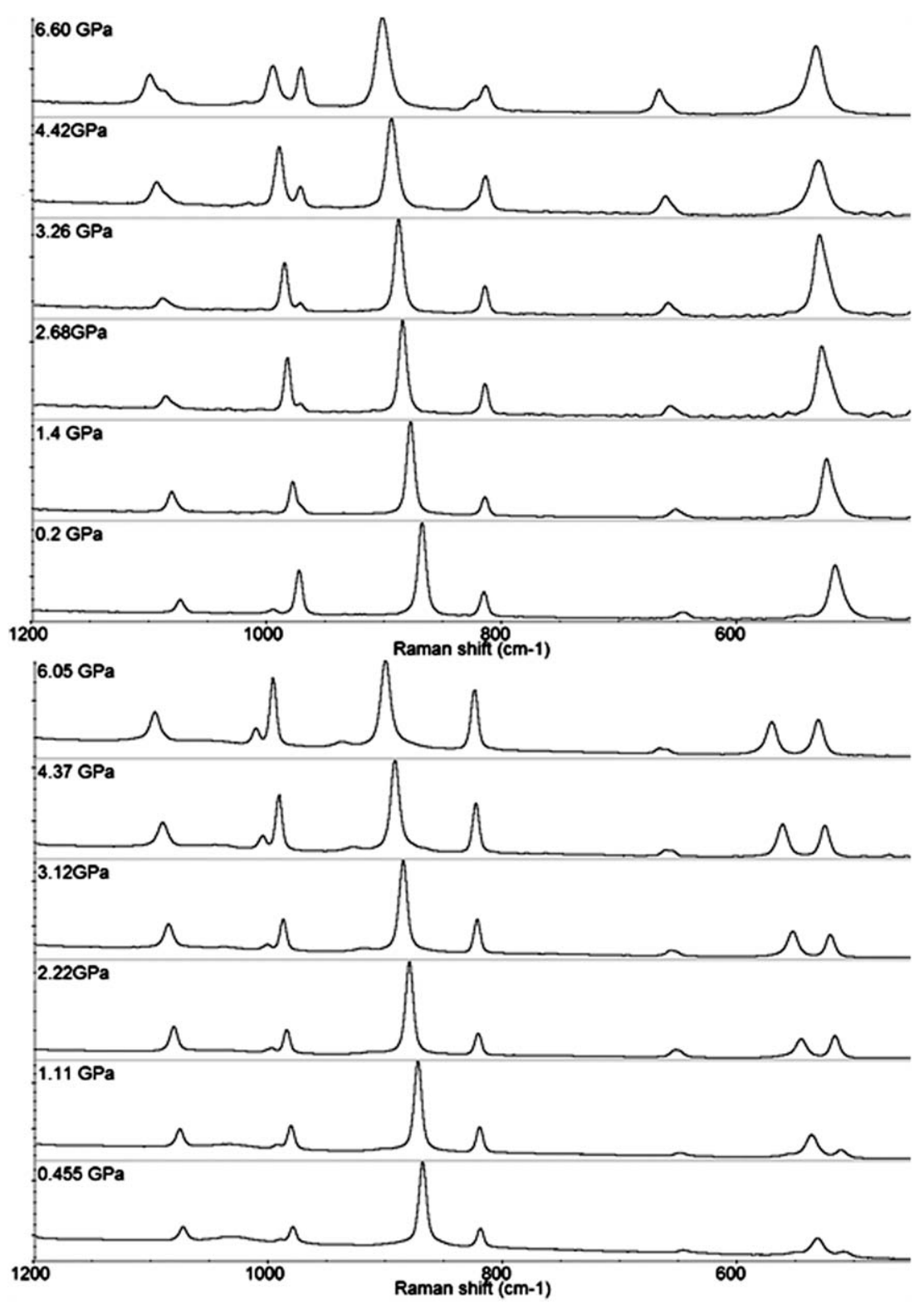

Fig. 2 The Raman spectra of Phase I (upper) and Phase II (lower) of acrylic acid on increasing pressure. Note the difference in the spectra between 500 and $550 \mathrm{~cm}^{-1}$.

The only further potential contact made between the columns is a long interaction between $\mathrm{C} 3 \mathrm{H} 3 \cdots \mathrm{O} 1$ which is beyond the sum of the van der Waals radii for the two atoms $\left(2.67 \AA\right.$ A, $\left.157^{\circ}\right)$.

During the high-pressure study of methacrylic acid we encountered a similar problem to the acrylic acid study where the melting point was too high for crystal growth from the melt and thermal polymerisation occurred. This prevented the growth of a new phase using the traditional techniques, however, it did give an insight into the behaviour of Phase I crystallised at high pressure. At $0.3 \mathrm{GPa}$, the cell parameters are longer than were found at $123 \mathrm{~K}$ which we found surprising given that, at least in the direction of the stacking, the separation between the molecules was greater than the lowtemperature form and the sum of the van der Waals for the two groups involved. One would have expected that this direction would have been compressed at this pressure. Comparison of the unit cell parameters obtained at high pressure with those collected at a temperature closer to ambient conditions ( $273 \mathrm{~K})$ showed a $\sim 4 \%$ reduction in volume with little change in the hydrogenbonding interaction that holds the dimers together (2.631(8) $\AA$ ). The interlayer distance between the dimer units is observed to be longer (3.54 ̊).

Following the successful isolation of a new polymorph of acrylic acid crystallised from the pressure-transmitting medium we set about trying to repeat this experiment with methacrylic acid. A $50: 50$ mixture of methacrylic acid and 4:1 methanol:ethanol was prepared and loaded into a DAC. The pressure was increased until the methacrylic acid crystallised from solution. A single crystal was grown through the modification of pressure and modest heating. At 1.5 GPa a new set of cell parameters were identified and a novel polymorph of methacrylic acid was structurally characterised. We 


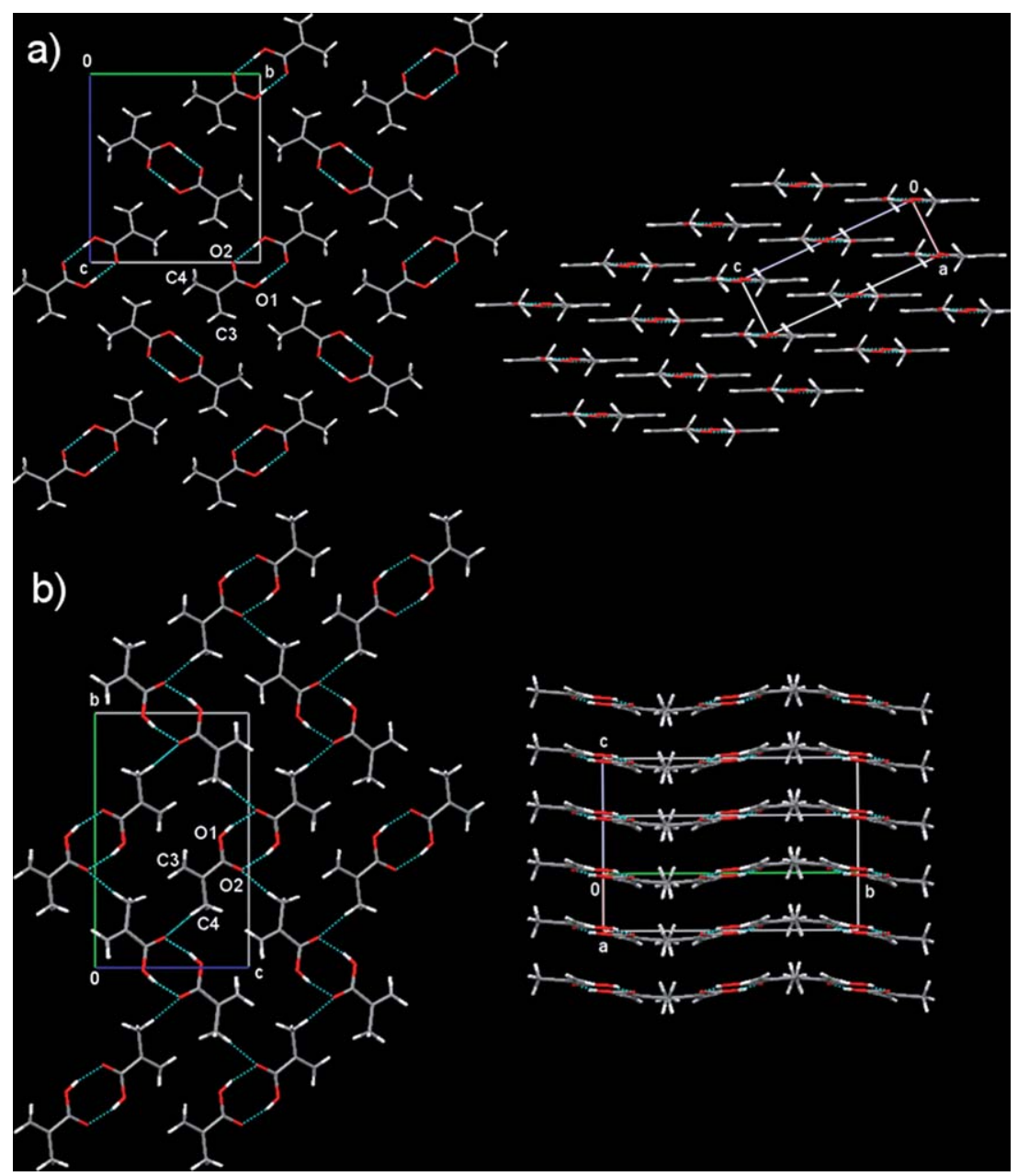

Fig. 3 The different packing arrangements in methacrylic acid at (a) $123 \mathrm{~K}$ and (b) $1.5 \mathrm{GPa}$. Red atoms—oxygen; grey atoms - carbon; white atoms— hydrogen.

shall designate this polymorph, Phase II. The crystallographic data can be found in Table S1†.

Fig. $3 b$ shows the interactions of the molecules within a layer of Phase II. As one can observe the dimer interactions remain and are

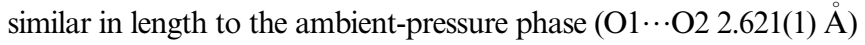
(Table S2 $\dagger$ ). The major change in this phase is the orientation of the neighbouring groups. In Phase I, the ethylene groups are positioned close to the carboxylic acid groups whereas in Phase II the methyl groups are observed to be close to the acid group $(2.43 \AA \mathrm{H} 5 \cdots \mathrm{O} 2$ normalised distance). Along with this change there is a rotation of the groups so that a corrugated plane of molecules is formed instead of the isolated dimers in Phase I with a reduction in mean plane separation to $3.19 \AA$ (Fig. $3 b$ cf. Fig. 3a).

The Raman spectra of the two phases are shown in Fig. 4 together with their response to pressure. There is a clear difference between the Raman spectra of the two phases. One can observe that in the region $\sim 400 \mathrm{~cm}^{-1}$ there are two bands present although in Phase II there is a slight shoulder at higher wavenumber on the peak at $370 \mathrm{~cm}^{-1}$. These bands are associated with the wagging vibration of the methyl and ethylene tail group $(\mathrm{C} 3 \mathrm{C} 2 \mathrm{C} 4)$ around the $\mathrm{C} 1-\mathrm{C} 2$ bond. More obvious difference lies in the region between 950 and $1010 \mathrm{~cm}^{-1}$ which describes the $\mathrm{CH}_{2}$ and $\mathrm{CH}_{3}$ rocking motion, respectively. ${ }^{12}$ In Phase
II the $950 \mathrm{~cm}^{-1}$ vibration is split further so that a $\mathrm{C}-\mathrm{C}$ stretching frequency of the methyl group is now observed as well as the wagging of the ethylene hydrogen atoms about the $\mathrm{C} 2-\mathrm{C} 3$ bond. $^{\mathbf{1 2}}$

Compression of methacrylic acid also induces the polymerisation despite the presence of the methyl group close to the ethylene group. ${ }^{13}$ Fig. $\mathrm{S} 1 \dagger$ shows the Raman spectrum for the polymer material produced from the direct compression of methacrylic acid downloaded to ambient pressure. One can observe that the spectra are very similar to one another suggesting that the product from the compression of either phase is the same. Acrylic acid also shows that the polymer produced from either polymorph is similar (Fig. S2 $\dagger$ ). In this case however there are peaks still present from some residual monomer $\left(1635 \mathrm{~cm}^{-1}\right)$.

In conclusion we have shown that acrylic and methacrylic acid form novel crystalline phases at high pressure. These structures are composed of the same dimer motifs that are present in the crystal structures determined at ambient pressure, however, the manner in which they are packed differs significantly. We have also shown that direct compression of either polymorph of acrylic or methacrylic acid can yield a polymeric substance. In fact, the Raman spectra show that a similar polymer can be obtained from either Phase I or Phase II of acrylic acid or methacrylic acid. The polymer that we have formed 


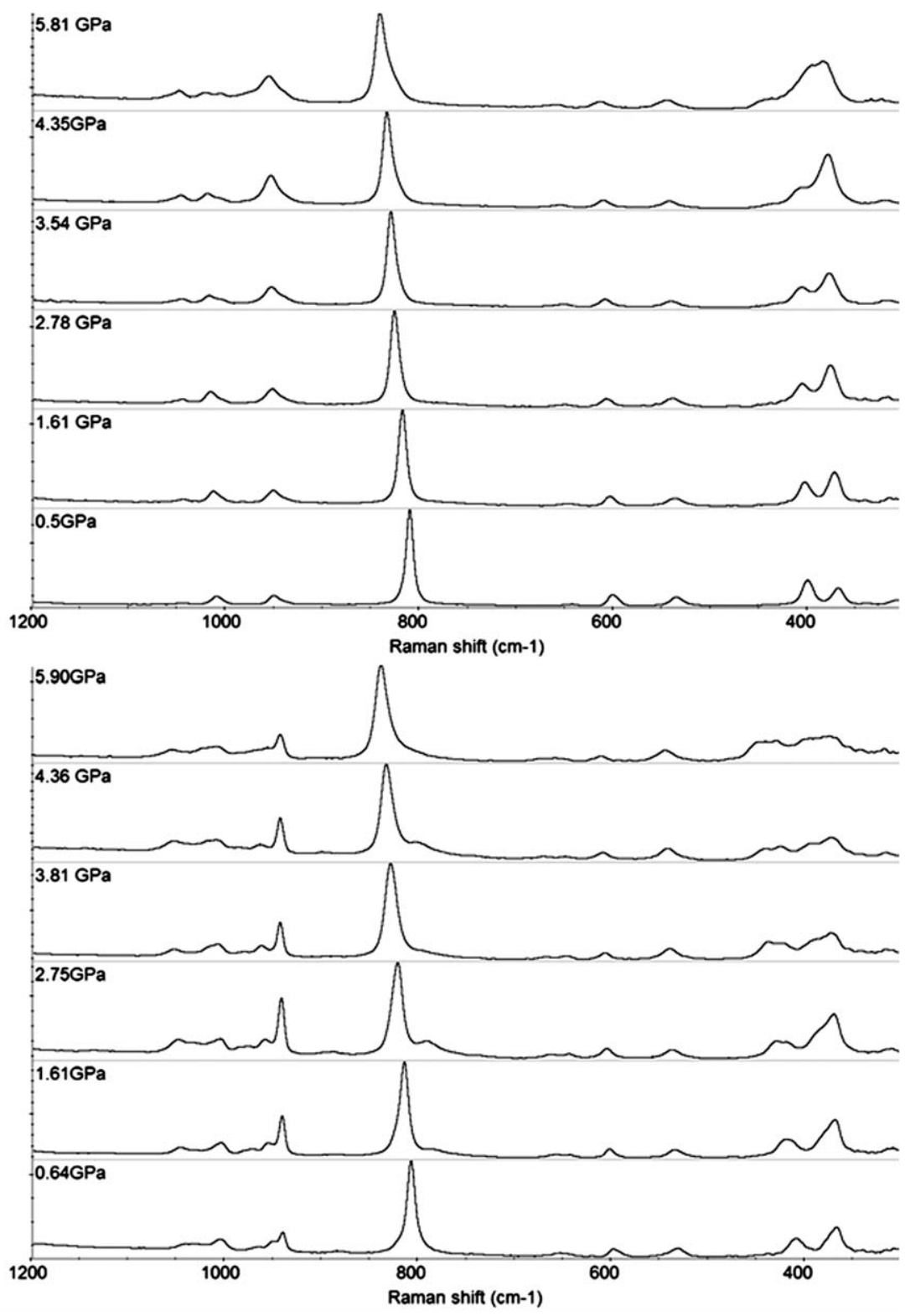

Fig. 4 The Raman spectra of Phase I (upper) and Phase II (lower) of methacrylic acid on increasing pressure.

from acrylic acid appears to be different to that isolated by Murli and Song in their paper.

\section{Acknowledgements}

The authors would like to thank Simon Parsons for the copies of the programs SHADE and Eclipse as well as the loan of the Diamond Anvil Cell. We would also like to thank the EPSRC for funding (EP/ E036244/1) (AJU).

\section{Notes and references}

1 M. Bradbury, S. Hamann and M. Linton, Aust. J. Chem., 1970, 23, 511.

2 D. Chelazzi, M. Ceppatelli, M. Santoro, R. Bini and V. Schettino, J. Phys. Chem. B, 2005, 109, 21658; M. Citroni, M. Ceppatelli, R. Bini and V. Schettino, J. Chem. Phys., 2005, 123, 9.
3 M. Ceppatelli, M. Santoro, R. Bini and V. Schettino, J. Chem. Phys, 2000, 113, 5991; M. Citroni, M. Ceppatelli, R. Bini and V. Schettino, Science, 2002, 295, 2058; M. Citroni, M. Ceppatelli, R. Bini and V. Schettino, J. Chem. Phys., 2005, 123, 194510.

4 C. Murli and Y. Song, J. Phys. Chem. B, 2009, 114(35), 9744.

5 M. A. Higgs and R. L. Sass, Acta Crystallogr., 1963, 16, 657.

6 R. Boese, D. Bläser, I. Steller, R. Latz and A. Bäumen, Acta Crystallogr., Sect. C: Cryst. Struct. Commun., 1999, 55, IUC9900006.

7 L. Merrill and W. A. Bassett, Rev. Sci. Instrum., 1974, 45, 290.

8 I. D. H. Oswald and W. A. Crichton, CrystEngComm, 2009, 11, 463.

9 G. Weck, P. Loubeyre and R. LeToullec, Phys. Rev. Lett., 2002, 88, 035504 .

10 A. Kulbida, M. N. Ramos, M. Rasanen, J. Nieminen, O. Schrems and R. Fausto, J. Chem. Soc., Faraday Trans., 1995, 91(11), 1571.

11 J. E. Davies and A. D. Bond, Acta Crystallogr., Sect. E: Struct. Rep. Online, 2001, 57, o947.

12 H. M. Badawi, M. A. A. Al-Khaldi, S. S. A. Al-Abbad and Z. H. A. AI-Sunaidi, Spectrochim. Acta, Part A, 2007, 68(3), 432.

13 S. Beuermann, M. Buback, P. Hesse and I. Lacik, Macromolecules, 2006, 39, 184-193. 